\title{
The determination of the single piece dispenser parameters
}

\author{
Evgeny Smekhunov ${ }^{1, *}$, Evgeny Chaika $^{1}$, and Irina Gritsay ${ }^{1}$ \\ ${ }^{1}$ Don State Technical University, Rostov-on-Don, Russia
}

\begin{abstract}
Automatic processing of fruits, root crops, single-piece vegetables is complicated by the need of single object supplying with the guaranteed intervals between them into the control zone. There is a need to create the simple, reliable single-piece giving piece dispensers. The information and the attention of researchers and at this issue is not enough. The questions of justification of the parameters substantiation of the conveyor single-piece dispenser are considered. The main conveyor speed positions, shaft diameters, profile configurations, the ways of setting a tuber on the conveyor, etc. are tested experimentally. The conclusion of the possibility and expediency of using the roller version of the single-piece dispenser is made.
\end{abstract}

\section{The setting of the problem}

One of the most effective means of increasing the labour productivity at manufacturing the labour-consuming products such as potatoes, vegetables, fruit, is a posthavesting processing automation: the lumpy impurity separation, non-conditionality products selection, sorting by the maturity, form and other quality features. An automatic control, as well as a manual one, is carried out by the inspection of each tuber or a fruit. At the same time, in our opinion, the capture of a potato by the manipulator and its transferring into the control zone is irrational. It is more practical to carry out an orderly product flow into the control zone and the division (into a single layer, into a single row, and then by the piece with the specified interval between tubers - a single piece dosing) by the mechanical device. Singlepiece dispensers are used to perform this operation.

The issues of a single-piece feeding have to be solved when designing a lot of machine types in various industries. For the correct-shaped products, which are damage-resistant, the problem is solved. However, for suppling of the agricultural products of various shapes and sizes, such as: potatoes, tomatoes and apples, a set of properties, which no dispenser has, is required. The analytical review showed that there are a number of single piece dispensers. Therefore, it is necessary to compare constructive decisions and to choose the most perspective option taking into consideration the set of positive and negative qualities. For the construction parametric optimization it is required to calculate the analytical conditions

\footnotetext{
*Corresponding author: smehunov@mail.ru
} 
of a single piece feeding of tubers out of the bulk mass and to implement this principle in a real construction.

\section{The last achievement and publication analysis}

To evaluate the constructions, the classification of dispensers based on the principle of action, execution the achieved result, was carried out before, that allowed to enlarge their analysis. According to the scheme, it is possible to visualize with which one of dispensers it is possible to divide into a single layer, into a row, into the pieces and, hence, to narrow the field of a rational design search.

According to the results of the information search of the single piece feeding mechanical devices it was found that for the nearest future the most perspective constructions are conveyor dispensers some of which have several foreign analogues of serial production [1-2].

Not all the dispensers are equally investigated and used. Some of them are considered by us at the level of the idea and their working off is still necessary.

Due to the development of design works on the separation automation, sorting, planting, the packaging of the single piece products of the definite weight it has become necessary to provide analytical equations for the basic parameters calculation of the single piece dispensers. Let's focus at one of the most productive and minimally affecting the product, conveyor dispensers [3-5].

The conveyor dispenser consists, at least, of two conveyors, the task of the first conveyor is to receive a product from the previous operation and previously to disperse the product, the task of the second conveyor is to fix the dispersed material and to feed it into the control zone. To arrange the bodies in one row on a horizontal surface, it is enough to give this surface vibration movement. The same result will be obtained if to incline a plane at an angle $\alpha 1$, which is greater than the angle of a tuber on a tuber rolling. The underlying potatoes will keep them from rolling down. The potatoes getting into the second layer of the inclined conveyor at the lower end will roll down. Further dispersion in the longitudinal direction occurs when moving from the conveyor to the conveyor due to the speed difference of the conveyor belts.

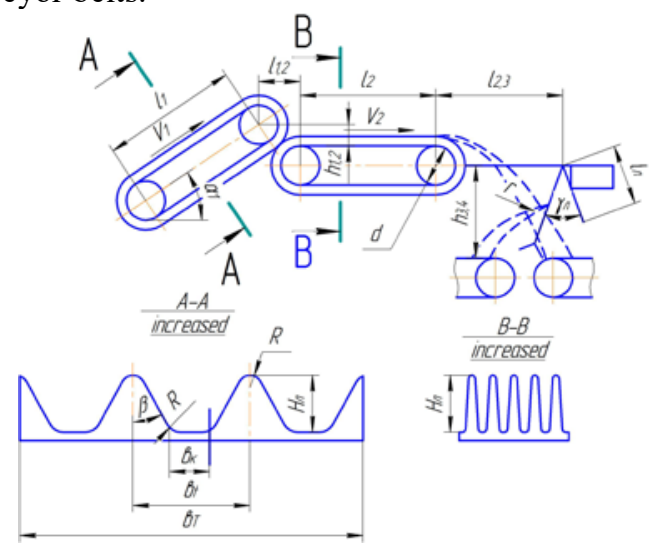

Fig. 1. Kinematic parameters of the dispenser system - an executive mechanism; $l_{1}, l_{2}$ - the length of the inclined and dosing conveyors; $l_{1,2}$ - the distance between conveyors; $l_{2,3}$ - the distance from the dosing conveyor to the executive mechanism upper hinge; $h_{1,2} h_{2,3} h_{3,4}$ - differences on height between conveyors and conveyors and the executive mechanism; $l_{A}$ - length of a shoulder blade, $H_{J}$ - the conveyor belt profile height; $b_{k}$ - the groove width of the conveyor belt profile; $b_{t}$ - the conveyor belt profile step; $\alpha_{1}$ the angle of the inclined conveyor installation; $\gamma_{J-}$ the shoulder blade deviation angle; $\beta$ - the angle of a cross section profile generatrix; $r$ - the trajectories dispersion; $d$ - the diameter of conveyor shafts. 
If it is necessary to provide feeding on rows the inclined conveyor with the $\mathrm{V}$ - shaped row walls is used (fig.1.).

We have determined productivity of the single layer $\mathrm{Q}_{1}$, slatted $\mathrm{Q}_{2}$, longitudinally inline $\mathrm{Q}_{3}$ and cellular $\mathrm{Q}_{4}$ dispensers.

If to take into account the parameters of the existing conveyors of the considered types, then their productivities have the following proportion:

$$
Q_{1}: Q_{2}: Q_{3}: Q_{4}=1: 0,35: 0,71: 0,51
$$

In a contact with a moving belt of the dosing transporter, the tubers some time make the movement relative to the belt. The minimum dispenser length will also be predetermined by the distance at which the process occurs - the alignment of a belt and a tuber speeds.

The V - shaped profile of the feeding conveyor grooves can be formed by the solid conveyor belt cut into plates (see the fig. 1, B-B) or by an elastic finger surface. In the latter case with the appropriate finger parameters, the alignment of a belt and a tuber speeds (a tuber damping) with the transporters speed difference up to $1 \mathrm{~m}$ occurs on the way, which isn't longer than $4 \mathrm{~cm}$, as it is defined below.

\section{The results of the discussion}

The condition of material transition from the conveyor to the conveyor is known [3]. For the material moving with the initial speed component coinciding with the speed of the second conveyor $V_{2}$, the speed $V_{k}$, which will be reached by a material particle at the length $l_{k}$ will be determined from the equation

$$
V_{k}=\sqrt{2 g * l_{k}\left(\mathrm{f} * \cos \alpha_{1} \sin \alpha_{1}\right)+\mathrm{V}_{0}},
$$

where $\alpha_{1}$ - a conveyor inclination angle; $f$ - the friction coefficient in couple a tuber (or a fruit) - a conveyor belt (in this case it is rubber).

In the equation (2) the significant factor - inertial forces, is not considered, but at $V_{0} \neq V_{2}$ there is an acceleration $\frac{V_{2}-V_{0}}{t_{2-0}}$ acting during $t_{2-0}$ of the speed alignment. So, it is possible to get the equation for $l_{k}$ provided that the tuber final speed $V_{k}$ is compared with the transporter speed $V_{2}$ :

$$
l_{k}=\frac{V_{0}\left(V_{2}-V_{0}\right)}{2 g\left(\mathrm{f} * \cos \alpha_{1}-\sin \alpha_{1}\right)}
$$

At the following data $\alpha_{1}=0, V_{0}=0,5 \mathrm{~m} / \mathrm{c}, V_{2}=1 \mathrm{~m} / \mathrm{c}, f=0,35$ is $l_{k}=0,036 \mathrm{~m}$.

Hence, after falling onto the dispenser second conveyor the potato tubers will roll back to $0.04 \mathrm{~m}$, this value determines the second conveyor working zone. In the experiments, the rollback - the way of tubers damping on the rubber finger-parted surface did not exceed 3 $\mathrm{cm}$, see [3].

The conveyor width is determined from the equation: 


$$
b_{T}=\frac{Q_{1-4}}{V_{1} * \bar{m} * n_{0}}
$$

where $\bar{m}$ - the tuber mass average value; $n_{0}$ - mass of tubers $\bar{m}$, arranged on in a single layer $1 \mathrm{M}^{2}$.

For values $Q=20 \mathrm{t} / \mathrm{h}(5.56 \mathrm{~kg} / \mathrm{s}), V_{1}=0,5 \mathrm{~m} / \mathrm{s}, \bar{m}=0,092 \mathrm{~kg}, b_{t}=0,075 \mathrm{~m}, b_{T}=$ $0.41 \mathrm{~m}$ it is received for a smooth belt, for a slatted apron dispenser $b_{T}=0.8 \mathrm{~m}$ for cellular $b_{T}=1.14 \mathrm{~m}$.

The similar differences in the transporter width in these limits are not so considerable as the number of other elements will not change.

The variation of the width and speed of the first conveyor will allow to obtain the required step of tubers feeding on the second conveyor, i.e. it will provide the dispersal.

Feeding in a single layer and in one row is feasible by the known adjusting devices. The implementation of tubers feeding one by one with the guaranteed intervals is still problematic. Earlier we calculated the general condition of bodies dispersal in the force field with the increasing gradient of the force vector [4-5]. The practically found solution is the use of the difference between the two conveyors which is a simple special case of the general condition of dispersion. Let's consider the movement of two tubers at a difference between feeding and dispersing conveyors at the inclination of the conveyors $\alpha_{1}$ and $\alpha_{2}$, diameters of shaft of $d$, speed $V_{1}$ and $V_{2}$, distances between shaft $l_{1,2} ; h_{1,2}$ (see the fig. 1), adjoining on the descent from the first conveyor. The location of a big tuber in front of a small tuber or two small ones will be harder conditions of tuber feeding, which reduce the time between their descents from the conveyor. The distance between their gravity centers is. $\frac{c_{1}+c_{2}}{2}=0,065-0,03 \mathrm{~m}$. где $c_{1}, c_{2}$ - the thickness of the nearby tubers.

The time between their descents from the conveyor is

$$
\Delta t=\frac{c_{1}-c_{2}}{2 V_{1}}
$$

In a period of time $t+\Delta t$ the distance between them will be

$$
e=\sqrt{\left(\mathrm{x}_{2}-\mathrm{x}_{1}\right)^{2}+\left(\mathrm{y}_{2}-\mathrm{y}_{1}\right)^{2}}=\frac{c_{1}-c_{2}}{2} \sqrt{1-\frac{\left(\mathrm{c}_{1}+c_{2}\right) \mathrm{g}^{2}}{4 V_{1}^{2}}\left(2 \mathrm{t}_{1}+\frac{c_{1}+c_{2}}{2 V_{1}}\right)^{2}}
$$

and, depending on the type of a separator or a sorter from 30 to $100 \mathrm{~mm}$ can be received.

The value $e$ is selected at the conditions of a necessary zone of the tuber control by the adjusting device, at the same time it must be kept in mind that the distance $e$ increases with the reduction $V_{1}$. From (4) it is solved, there can be also the return task can also be solved, the time, $t_{1}$ in which the bodies will appear at necessary distance of $e$. 
At $V_{1}=0.25 \mathrm{~m} / \mathrm{s}, t_{1}=0.2 \mathrm{~s}$ and $\frac{c_{1}+c_{2}}{2}=0.03 \mathrm{~m}$ we will receive $e=0.06 \mathrm{~m} l_{1,2} ; h_{1,2}$ respectively 0.05 and $02 \mathrm{~m}$. Having determined $e$ from (4), we will find the distance between the next falling tubers:

$$
t_{i}=e-\frac{c_{1}+c_{2}}{2}
$$

It is possible to determine the distance between conveyors $h_{3,4}$ based on a condition that the horizontal component of speed of falling of the subsequent body is less, than the speed of a tape of the second conveyor $V_{2}$. Otherwise there will be an impact of bodies. This condition has the following appearance:

$$
V_{2} \succ \sqrt{\left(V_{1}^{2}+2 g h_{3,4}\right) \cos \zeta}
$$

where $\zeta$ - the angle of the trajectory inclination to the horizon at the point of intersection with the horizontal dosing transporter.

$$
h_{3,4} \prec \frac{\frac{V_{2}^{2}}{\cos \xi}-V_{1}^{2}}{2 g}
$$

i.e. at $V_{2} \succ V_{1}$ impacts will not be.

If two tubers are nearby, then time between a descent of the first and second tubers is $\Delta t$.

The same time will pass between their falling onto the second conveyor. The first tuber moving with a belt speed $V_{2}$ will pass the distance from the contact place

$$
s_{T}=V_{2} \frac{c_{1}+c_{2}}{2 V_{1}}
$$

The more there will be a difference between $V_{1}$ and $V_{2}$ the more there will be a feeding step therefore $V_{1}$ it is desirable to choose with smaller values, trying to obtain the increase in productivity at the expense of the conveyor width $b_{T}$.

At $V_{1}=0,25 \frac{c_{1}+c_{2}}{2}=0,03 \mathrm{~m}, V_{2}=0,75 \mathrm{~m} / \mathrm{s}$ the distance between tubers taking into account (5) is $0.045 \mathrm{~m}$. As a result of the calculations the parameters necessary for designing of single piece feeding various dispensers of various agricultural products can be determined.

Experimental definition of a way of calm of a fruit upon transition from the conveyor to the $l_{k}$ conveyor allows to turn the single piece conveyor dispenser of tubers into a roll (fig.2) one at the same parameters of the feeding surface. Such a dispenser even at three- 
four rollers will have dimensions less, than those of a conveyor dispenser and considering that $\mathrm{V} 3>\mathrm{V} 2>\mathrm{V} 2$, the dispersal range will be greater.

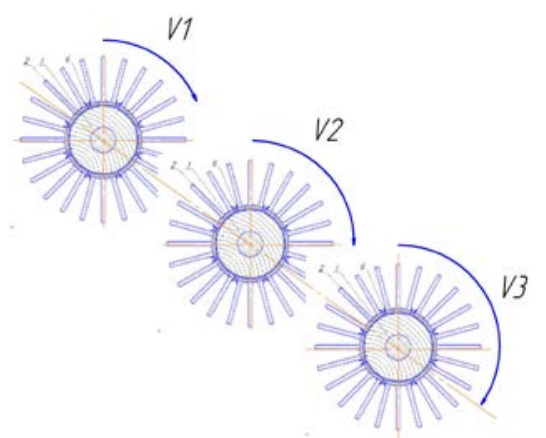

Fig. 2. The single piece dispenser of potatoes in the rolling construction.

\section{References}

1. R.H.Yusupov, S.A. Andreyev, The robotic manipulators usage for piece feeding and movement of agricultural products, Federal public educational institution of higher and professional training Bulletin "Moscow State Agro engineering University after V.P. Goryachkin" No. 3, (2017)

2. Han, Yang, W.b, Zhang, X.b, Guo, H.b, Yin, W.a Design and test of the automatic feed system for tray seedlings transplanter, Nongye Gongcheng Xuebao/Transactions of the Chinese Society of Agricultural Engineering v. 29, pp.51-61 (2013)

3. G.D. Petrov, Potato-harvesting machines, Mechanical engineering, pp.262-268 (1972)

4. N.N. Kolchin, E.A. Smekhunov, V.M. Lyamenkov, The single-piece feeding device for piece items, mainly potato tubers, to the sorting device, Inventor's certificate, 596519 (The USSR) B.I., No. 9 (1978)

5. E.A. Smekhunov, The determination of single piece transporter parameters, RIACM Rostov-on-Don (1984) 